\title{
Multi-frame Image Processing Based on Wireless Sensor Networks
}

\author{
https://doi.org/10.3991/ijoe.v14i10.9306 \\ Xianfeng Yang $(\bowtie)$, Xiaojian Jia \\ Henan Institute of Science and Technology, Xinxiang 453003, China, \\ 49377535 eqq. com
}

\begin{abstract}
With multi-frame image processing based on wireless sensor network as research object, this study analyzes the problems existing in the two classic multi-node collaborative distributed image processing algorithms based on wavelet transform using literature analysis, simulation experiment, data analysis and other research methods, proposes a distributed image compression algorithm based on SHIHT with the application of wireless sensor network, SPIHT algorithm and wavelet transform to image compression as theoretical foundations, and adopts the matrix laboratory to simulate the energy performance of the algorithm. Through comparative analysis, the feasibility of distributed image compression algorithm based on SHIHT presented in this study is verified.
\end{abstract}

Keywords-Wireless Sensor, Image Processing, Distributed Image Compression, Wavelet Transform

\section{Introduction}

Image is the visual expression of an object through the eyes, and is one of the major ways to obtain outside information [1], which plays a remarkable role in environmental monitoring, military applications, medical care and other fields. In view of the huge image data amount, it is necessary to compress the data while ensuring the image quality in order to guarantee a high speed in the transmission process.

Wireless sensor network (WSN), a distributed sensor network that can inspect and sense the external world, can realize the functions of data collection, processing and transmission, which belongs to a special form of multi-hop self-organizing network [2]. In the process of image acquisition and transmission, especially when the amount of acquired image information is large, distributed image compression technology is particularly important, as the large bandwidth and energy consumption of each node would lead to the low efficiency of data collection and transmission.

Distributed image compression technology is one of the hot topics in wireless sensor network studies, whose main idea [3] is to reduce the amount of wireless transmission data by compressing data at sensor nodes, then the compressed data is sent to the convergence node in a multi-hop manner, which will be decompressed when used. However, in the conventional centralized compression algorithms, the processing capacity of a single sensor node is limited, the energy is easily exhausted, and the network life 
cycle is short [4]. The general distributed compression algorithm can improve the life cycle of wireless sensor networks to a certain extent, but its energy consumption distribution is uneven and the energy consumption of wireless transceiver is too large.

To sum up, based on the elaborations of the application of wireless sensor network, SPIHT algorithm, and wavelet transform to image compression, this study analyzes the problems existing in two classic multi-node collaborative distributed image processing algorithms based on wavelet transform, proposes a distributed image compression algorithm based on SHIHT, designs its processing structure in detail and finally obtains experimental data through matrix laboratory simulation. The data are analyzed from the aspects of the relationship between node energy consumption and the single-hop average distance, the relationship between total energy consumption and hop number, and the relationship between network life cycle and node density, which verifies the feasibility of the proposed SHIHT-based distributed image compression algorithm.

\section{Research on Distributed Image Compression Algorithms for Wireless Sensor Networks}

Wireless Sensor Network (WSN), a distributed sensor network that can inspect and perceive the external world, can realize the functions of data collection, processing, and transmission, which is composed of processor, sensor, wireless communication and energy supply [5].

Table 1. WSN network development

\begin{tabular}{|c|l|l|}
\hline Time & \multicolumn{1}{|c|}{ Name } & \multicolumn{1}{c|}{ Sensor network development } \\
\hline $70 \mathrm{~s}$ & First generation sensor network & Point-to-point transmission \\
\hline $80 \mathrm{~s}$ & Second generation sensor network & Ability to comprehensively process information \\
\hline $90 \mathrm{~s}$ & Third generation sensor network & Fieldbus technology is applied \\
\hline to date & Fourth generation sensor network & Data-centric, self-organizing sensor networks \\
\hline
\end{tabular}

Table 1 shows the history of WSN development [6]. The fourth generation sensor network, also known as wireless sensor network, has been widely used in military, medical, environmental monitoring and other fields. Distributed image compression and data fusion technologies among others are the key technologies for wireless sensor networks [7].

\subsection{Application of wavelet transform in image compression}

The wavelet transform mainly uses the locality and directionality after transformation to achieve the application in image compression, which belongs to the multiresolution analysis method [8]. It contains two parts, a high frequency subband which occupies a small part of energy and a low frequency subband that occupies most of the energy [9]. The image transformed with wavelet transform has higher compression ratio and can better preserve the original image quality. Figure 1 shows the wavelet transform image compression and decoding process [10]. 


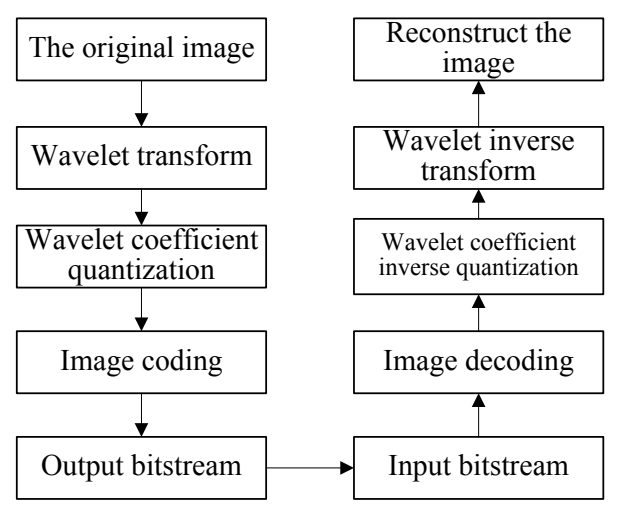

Fig. 1. Wavelet transform image compression and decoding process

\subsection{Multi-node collaborative distributed image processing algorithm based on wavelet transform}

The image distributed compressed encoding method can reduce the energy consumption of the compression processing of a single node, integrate the resources of each node, and improve the network life cycle without increasing the network cost [11]. The realization of a multi-node collaborative distributed image processing algorithm requires the cooperation and exchange of sensor nodes. The two classic multi-node collaborative distributed image processing algorithms based on wavelet transform are shown in Figure 2 [12], both of which adopt the clustering structure commonly used in sensor networks. Through the analysis of the two algorithms, it can be found that although the algorithm b) can effectively reduce the energy consumption and improve the compression effect compared with the algorithm a), both algorithms have the large energy consumption of wireless transmission between nodes, and unbalanced node energy consumption [13-14].

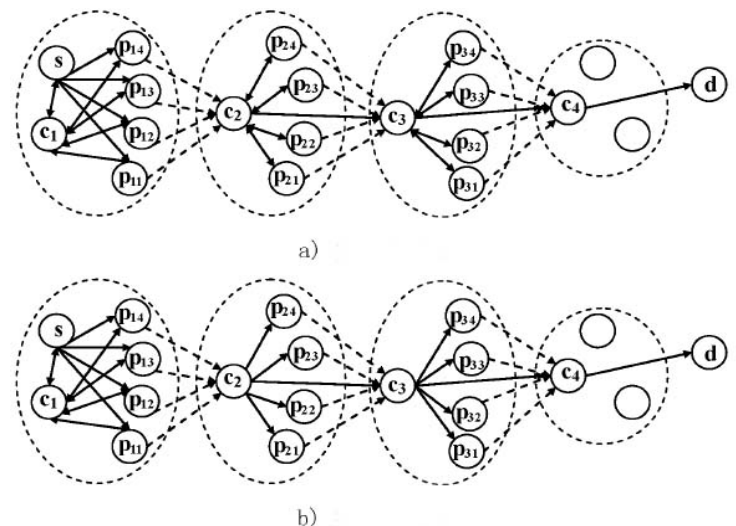

Fig. 2. Multi-node collaborative distributed image processing algorithm based on wavelet transform 


\section{Design of Distributed Image Compression Algorithm Based on SHIHT}

\subsection{Analysis of SPIHT algorithm}

Embedded zero-wavelet coding (EZW), set partitioning in hierarchical trees (SPIHT), and embedding block coding algorithm with optimized truncation points (EBCOT) are three classic compression algorithms for images based on wavelet transform [15], which make full use of the multiresolution nature of wavelet transforms. The SPIHT algorithm is an improvement over the EZW algorithm, which, unlike the EZW algorithm, defines a spatial direction tree structure [16]. As shown in Figure 3, the wavelet pixels of each node are represented by coordinates, which effectively improves the encoding efficiency.

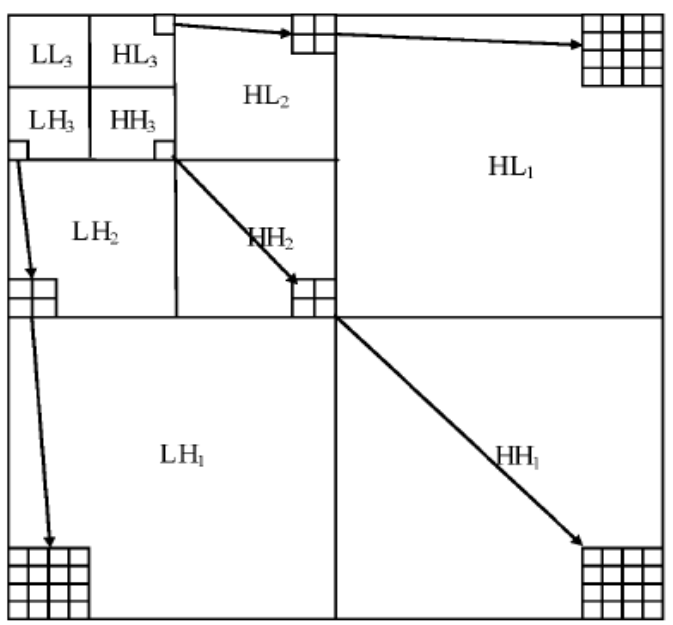

Fig. 3. Spatial Directional Tree Structure of SPIHT Algorithm

The SPIHT coding needs to record the coded set of nodes through three linked lists: list of insignificant pixels (LIP), list of significant pixels (LSP) and list of insignificant sets (LIS). Application of SPIHT encoding is divided into four processes: initialization, sequential scanning, refine scan and next round of sequential scanning and refine scan [17]. 


\subsection{Design of distributed image compression algorithm based on SHIHT}

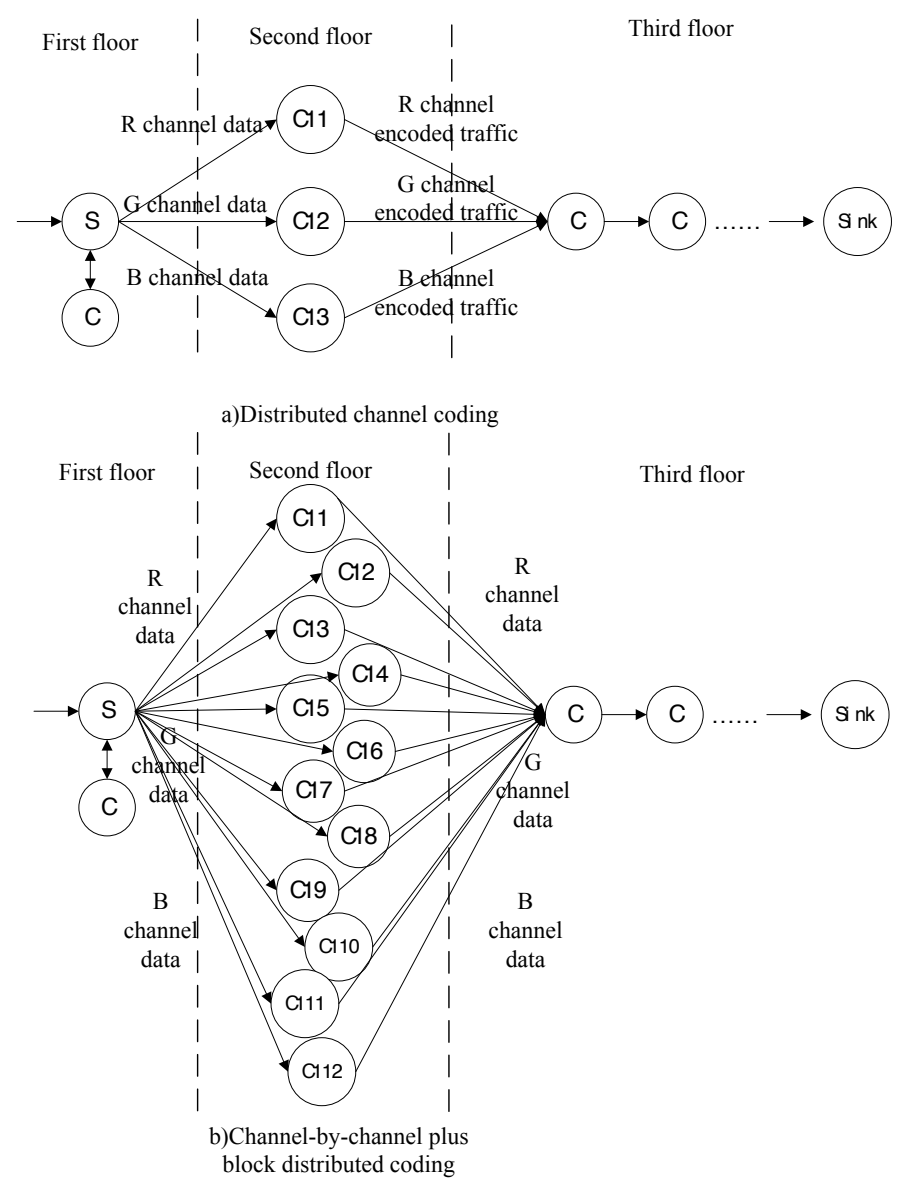

Fig. 4. Distributed processing architecture

Inspired by the multi-node collaborative distributed image processing algorithm and SHIHT algorithm based on wavelet transform, a distributed image compression algorithm based on SHIHT is proposed in this study, which can perform distributed channel compression on color images. Figure 4 shows the SHIHT-based distributed image compression algorithm processing architecture [18]. The structure consists of three layers of data streams. The $\mathrm{S}$ nodes and the cluster head node $\mathrm{Ci}$ responsible for collecting and processing the images in separate channels form the first layer of data streams. The second layer consists of $C_{i j}$ nodes that are responsible for receiving images and performing SHIHT compressed encoding. The images are compressed and sent to the next layer of nodes through this layer. The third layer of data stream is composed of the cluster head nodes of the next layer, whose function is to send the encoded stream of the summary encoded processing node to the sink node in a multi-hop manner. 


\subsection{Simulation of energy consumption performance of the algorithm}

(1) Energy consumption model

Data acquisition, transceiving and processing are major parts of the energy consumption of wireless sensor networks. There is little difference in energy consumption of data acquisition, thus can be ignored. The differences in energy consumption of the latter two are mainly considered.

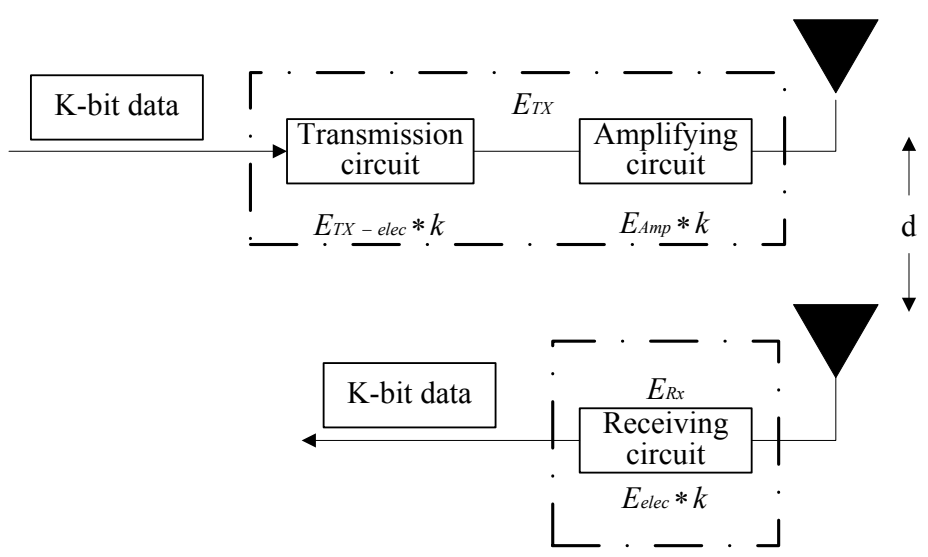

Fig. 5. Sensor wireless transceiver FORM energy consumption model

Figure 5 shows a common energy consumption model of wireless sensing network radio, in which the receiving module (receiving circuit) and the transmitting module are the main energy consumption points of the sensor nodes. The energy consumption per bit of data $(\mathrm{k})$ at the receiving terminal and at the transmitting terminal can be determined by the following formula:

Sender : $E_{T X}=E_{T X}-$ elec $+E_{\text {amp }}$

Receiver: $E_{R x}=E_{R x}-$ elec

Where, $E_{T X-e l e c}$ and $E_{R X-e l e c}$ represent the received and transmitted energy consumption per unit of bit, and $E_{a m p}$ represents the energy consumed by the transmitting terminal when sending data through the amplifier, which is proportional to the fourth power of distance d, i.e.:

$E_{a m p}=\left\{\begin{array}{l}\varepsilon_{f s} \times d^{2}, d \leq d_{0} \\ \varepsilon_{m p} \times d^{4}, d>d_{0}\end{array}\right.$

According to the transmission distance, choose $E_{a m p}=\varepsilon_{f s} \times d^{2}$, then

$$
E_{T X}=E_{T X}-\text { elec }+\varepsilon_{f s} \times d^{2}
$$

Energy consumption of image processing includes two parts: quantization coding $\left(E_{\text {code }}\right)$ and wavelet decomposition energy consumption $\left(E_{c o d e}\right)$, that is. 
$E_{\text {comp }}=E_{\text {DMT }}+E_{\text {Code }}$

(2) Performance simulation analysis

With the proposed distributed algorithm and the SPIHT algorithm based on wavelet transform, this study selects the color images with a resolution of $\mathrm{M} \times \mathrm{N}$ and 8 bits per pixel for matrix laboratory simulation, through which the relevant experimental data are obtained. The energy consumption of each node is analyzed as follows:

Collect energy consumption of node $\mathrm{S}: E_{\mathrm{s}}=M \times N \times 8 \times 3 \times E_{T X}$

Processing node $\mathrm{C}_{\mathrm{ij}}$ energy consumption: $E_{\mathrm{Cij}}=\left(M \times N \times 8 \times \mathrm{ERx}_{\mathrm{Rx}}+M \times N \times 8 \times E_{T X} / r+M \times N \times 8 \times E_{\text {comp }}\right) / 4$

Receive node C energy consumption : $E_{\mathrm{C}}=M \times N \times 8 \times 3 \times\left(E_{T X}+E_{\mathrm{Rx}}\right) / \mathrm{r}$

Distributed system total energy consumption : $E_{1}=E_{\mathrm{s}}+E_{\mathrm{p}} \times 4 \times 3+E_{\mathrm{C}}$

(5)

If a centralized processing method is adopted, wherein the images at node $\mathrm{S}$ are first collected and compressed and then transmitted, then the energy consumption at node $\mathrm{S}$ is:

$E_{2}=M \times N \times 8 \times 3 \times E_{\text {comp }}+M \times N \times 8 \times 3 \times E_{T X} / r$

Analysis of relationship between node energy consumption and single-hop average distance. Table 2 shows the relationship between energy consumption and single-hop transmission distance in distributed and centralized processing acquisition nodes when the compression ratio is 8 .

The energy consumption at each node in the code cluster increases with the increase of single-hop distance. The energy consumption at node $\mathrm{S}$ is the most obvious, while the energy consumption at encoding node $\mathrm{Cij}$ is the least affected by the single-hop distance. This is because the images acquired at the node $\mathrm{S}$ are uncompressed thus the data amount is huge, and that the increase in energy consumption is proportional to the square of the distance. However, after the distributed structure distributes the compression tasks evenly, the compressed encoding reduces the data transmission volume, thus the data processing capacity of the encoding node $\mathrm{Cij}$ decreases. Through the above analysis, in the distributed network structure, the node used for encoding processing should be set near the node $\mathrm{S}$ so as to reduce the transmission volume and reduce the energy consumption.

The total energy consumption of distributed compression is higher compared to centralized compression. When the single-hop distance is small, the energy consumption among nodes is not much different, and the $\mathrm{S}$ node consumes the most energy. However, the energy consumption of centralized compression is all concentrated on node $\mathrm{S}$, and when the energy of centralized compression is exhausted, the whole compression structure network will fail. Therefore, compared with the distributed compression structure, when the distance is small, the integrated compression structure has fast energy consumption and short network life cycle.

When the single-hop distance is greater than $70 \mathrm{~m}$, compared with the integrated compression structure, the distributed compression structure has larger total energy 
consumption and a shorter life cycle, thus does not have much advantages. However, due to the fact that sensor network nodes are usually arranged at a distance of less than $70 \mathrm{~m}$, the use of a distributed system at this time is conducive to extending the life cycle of the sensor network.

Table 2. Node energy consumption and single-hop transmission distance (J)

\begin{tabular}{|c|c|c|c|c|c|}
\hline Single-hop distance & S node & Cij node & C node & Total energy consumption & Centralized \\
\hline $5 \mathrm{~m}$ & 0.3302 & 0.2941 & 0.0805 & 3.9441 & 3.2184 \\
\hline $10 \mathrm{~m}$ & 0.3776 & 0.2048 & 0.0862 & 4.0027 & 3.2245 \\
\hline $20 \mathrm{~m}$ & 0.5661 & 0.2968 & 0.1100 & 4.2388 & 3.2479 \\
\hline $30 \mathrm{~m}$ & 0.8807 & 0.3301 & 0.1495 & 4.6322 & 3.2872 \\
\hline $40 \mathrm{~m}$ & 1.3211 & 0.3046 & 0.2044 & 5.1827 & 3.3422 \\
\hline $50 \mathrm{~m}$ & 1.8875 & 0.3105 & 0.2752 & 5.8903 & 3.4130 \\
\hline $60 \mathrm{~m}$ & 2.5794 & 0.3175 & 0.3678 & 6.7556 & 3.4997 \\
\hline $70 \mathrm{~m}$ & 3.3975 & 0.3265 & 0.4641 & 7.7779 & 3.6018 \\
\hline $80 \mathrm{~m}$ & 4.3412 & 0.3361 & 0.5819 & 8.9576 & 3.7199 \\
\hline
\end{tabular}

Analysis of the relationship between total energy consumption and hop number. With the transmission of a $512 \times 512$ color image as an example, and with a singlehop average distance of $10 \mathrm{~m}$, and a range of 1 to 15 hops, this study compares the total energy consumption required per bit for centralized SPIHT, distributed SPIHT compression, and direct transmission. As shown in Figure 6 , since the uncompressed images in the distributed structure need to be transmitted to the encoding nodes for transmission through the acquisition node, the total energy consumption of this structure is higher when the compression ratio is the same compared to that of the centralized structure. This result is the same as the result shown in Table 2, but for this reason, the distributed node reduces the energy consumption of the acquisition node, thereby increasing the life cycle of the network. The energy consumption of the direct transmission image increases linearly and rapidly with the increase of the number of hops, while in the integrated and distributed structure, the initial value of energy consumption is high, and then linearly increases, with a low slope, indicating that the total energy consumption of compression and transmission increases with the increase of the number of hops. 


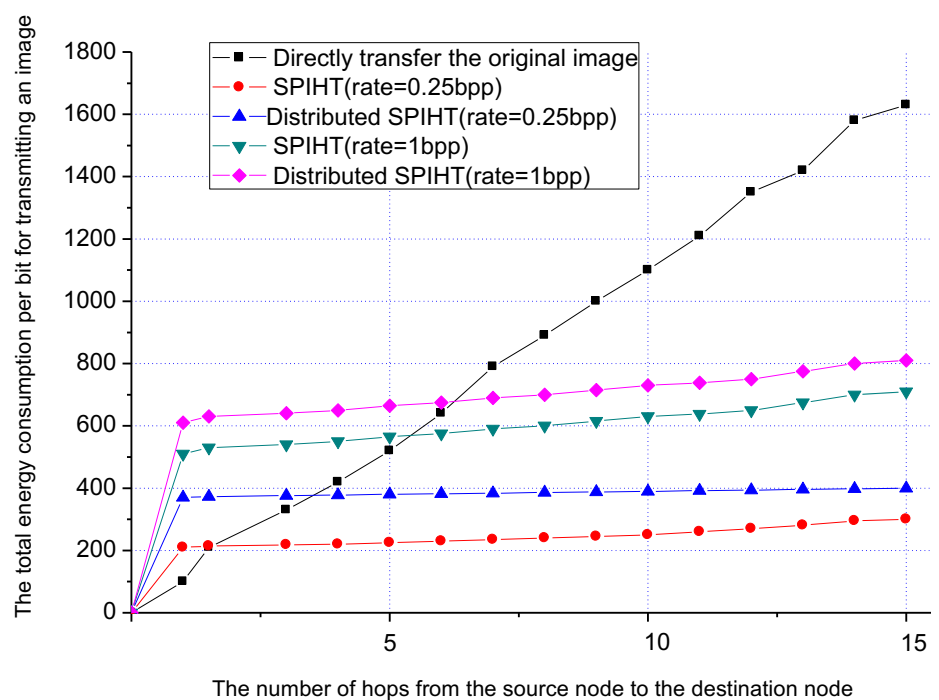

Fig. 6. Comparison of the total energy consumption per bit for transmitting an image

Relationship between network life cycle and node density. It is assumed that a different number of sensor nodes with an initial energy of 10 joules are arranged in a $200 \times 200$ area. When the energy is exhausted, the network life cycle ends. The comparison between the integrated model and the proposed SPIHT distributed structure algorithm is conducted.

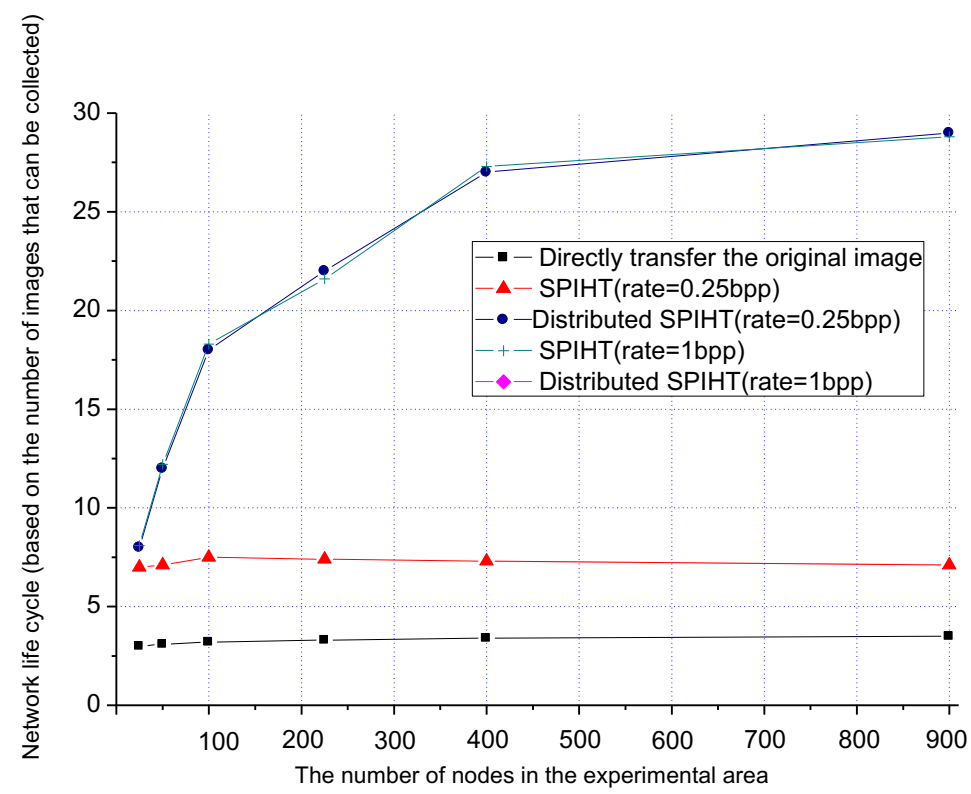

Fig. 7. Distributed and centralized structure network life cycle comparison 
Figure 7 shows the comparison of network life cycle between distributed and centralized structures, from where it can be seen that the life cycle of the distributed structure network increases with the increase of the number of nodes in the area, and the influence of the reduction of compression ratio on the life cycle of the network can be neglected. This is because the acquisition node in the distributed structure is the node with the largest energy consumption, thus the energy consumed by compressed encoding is not reflected in it, but the increase of node number has little effect on the life cycle of the integrated structure, and that its life cycle also decreases to a certain extent with the decrease of compression ratio.

\section{Conclusions}

Based on the references at home and abroad, this study discusses the distributed image compression technology based on wireless sensor network in depth, with the multiframe image processing method based on wireless sensor network as the research object. The specific conclusions are as follows.

1. A distributed image compression algorithm based on SHIHT is proposed in light with the analysis of the problems existing in two classic multi-node collaborative distributed image processing algorithms based on wavelet transform.

2. The structure of the distributed image compression algorithm based on SHIHT is designed in detail, which divides the color image into blocks or channels for processing.

3. Through the simulation experiment of energy consumption performance of the algorithm, it is verified that the SHIHT-based distributed image compression algorithm proposed in this study can effectively reduce energy consumption and extend the network life cycle.

\section{$5 \quad$ References}

[1] Razzak, M. I., Khan, M. K., Alghathbar, K., Park, J.H. (2011). Energy efficient distrib-uted face recognition in wireless sensor network. Wireless Personal Communications, 60(3): 571582. https://doi.org/10.1007/s11277-011-0310-y

[2] Pham, D. M., \& Aziz, S. M. (2013). Object extraction scheme and protocol for energy efficient image communication over wireless sensor networks. Computer Networks, 57(15): 2949-2960. https://doi.org/10.1016/j.comnet.2013.07.001

[3] Phat, N. H., \& Vinh, T. (2010). Low-complexity and energy-efficient algorithms on image compression for wireless sensor networks. Ieice Transactions on Communica-tions, 93B(12): 3438-3447.

[4] Ye, S., Lin, Y., Yi, Y. (2011). Energy-aware interleaving for robust image transmission over visual sensor networks. Iet Wireless Sensor Systems, 1(4): 267-274. https://doi.org/10.1049/iet-wss.2011.0050

[5] Yen, Y. S., Hong, S., Chang, R.S., Chao, H.C. (2009): Controlled deployments for wire-less sensor networks. Iet Communications, 3(5), 820-829. https://doi.org/10.1049/ietcom.2008.0262 
[6] Nasri, M., Helali, A., Sghaier, H., Maaref, H. (2012). Trade-off analysis of energy consumption and image quality for multihop wireless sensor networks. International Journal of Wireless Information Networks, 19(3), 254-269. https://doi.org/10.1007/s10776-012-0174$\underline{4}$

[7] Aziz, S. M., \& Pham, D. M. (2013). Energy efficient image transmission in wireless multimedia sensor networks. IEEE Communications Letters, 17(6): 1084-1087. https://doi.org/10.1109/LCOMM.2013.050313.121933

[8] Kim, S.P., Su, W.Y. (1993). Recursive high-resolution reconstruction of blurred mul-tiframe images. IEEE Transactions on Image Processing, 2(4): 534-539. https://doi.org/10.1109/ 83.242363

[9] Ozkan, M.K., Erdem, A.T., Sezan, M.I., Tekalp, A.M. (1992). Efficient multiframe wie-ner restoration of blurred and noisy image sequences. IEEE Transactions on Image Processing, 1(4): 453-476. https://doi.org/10.1109/83.199916

[10] Zhou, Y., Xiang, W., Wang, G. (2015). Frame loss concealment for multiview video transmission over wireless multimedia sensor networks. IEEE Sensors Journal, 15(3), 1892-1901. https://doi.org/10.1109/JSEN.2014.2366511

[11] Farsiu, S., M.D. Robinson, Elad, M., Milanfar, P. (2004). Fast and robust multiframe super resolution. IEEE Transactions on Image Processing, 13(10): 1327-1344. https://doi.org/10.1109/TIP.2004.834669

[12] Horio, T., Suzuki, T. (2009). Multihit two-dimensional charged-particle imaging sys-tem with real-time image processing at 1000 frames/s. Review of Scientific Instru-ments, 80(1): 013706. https://doi.org/10.1063/1.3062945

[13] Showail, A., Elrasad, A., Meer, A., Daghistani, A., Jamshaid, K., Shihada, B. (2014). Ifrag: interference-aware frame fragmentation scheme for wireless sensor networks. Wireless Networks, 20(7): 2019-2036. https://doi.org/10.1007/s11276-014-0722-1

[14] Fedali, S., Madani, H. (2017). Azeotropic points with relative volatility-prediction and calculation. Mathematical Modelling of Engineering Problems, 4(1): 38-42. https://doi.org/10.18280/mmep.040108

[15] Reddy, R.K., De, S., Gupta, H.M. (2010). Joint control of transmit power and frame size for energy-optimized data transfer in wireless sensor networks. IEICE Transac-tions on Communications, 93(8): 2043-2052. https://doi.org/10.1587/transcom.E93.B.2043

[16] Cordeiro, P. J., Assunção, P. (2012). Distributed coding/decoding complexity in video sensor networks. Sensors, 12(3), 2693-709. https://doi.org/10.3390/s120302693

[17] Lloret, J., Bosch, I., Sendra, S., Serrano, A. (2011). A wireless sensor network for vine-yard monitoring that uses image processing. Sensors, 11(6): 6165-6196. https://doi.org/10.3390/ s110606165

[18] Zhulina, Y.V. (2006). Multiframe blind deconvolution of heavily blurred astronomi-cal images. Applied Optics, 45(28): 7342-7352. https://doi.org/10.1364/AO.45.007342

\section{Authors}

Xianfeng Yang received his bachelor's degree in Computer Application Technology from Henan Normal University, Xinxiang, Henan, China, in 2001, the master's degree in Computer Application Technology from China University of Petroleum, Dongying, China, in 2007. He is now an associate professor at School of Information Engineering, Henan Institute of Science and Technology, Xinxiang, China. His current research interests include pattern recognition, image processing, neural networks, natural language processing. 
Xiaojian Jia received her bachelor's degree in Information Engineering from Henan University of Science and Technology, Luoyang, Henan, China, in 2009, the master's degree in Communication and Information System from Shandong University, Jinan, China, in 2013. She is now an assistant at School of Information Engineering, Henan Institute of Science and Technology, Xinxiang, China. Her current research interests include image processing, pattern recognition, wireless resource optimization and Cognitive radio network.

Article submitted 15 August 2018. Resubmitted 23 September 2018. Final acceptance 18 October 2018. Final version published as submitted by the authors. 